\title{
Purulent pericarditis followed by early constriction in young children
}

\author{
R. Caird, N. Conway, and I. K. R. McMillan \\ From The Wessex Regional Cardiac and Thoracic Centre, Western Hospital, Southampton
}

Two young children with acute purulent pericarditis are described, and the management of this condition is discussed. Open pericardial drainage is necessary as well as antibiotics. Constriction may follow very rapidly and occurred in both patients. It was successfully treated in one of them, requiring epicardial as well as pericardial removal for relief.

Two children have recently been seen with acute purulent pericarditis. In both early constriction followed and one survived after extensive pericardectomy.

\section{Case reports}

Case I

A 28-month-old Negress was admitted to the Middlesex Hospital with acute bronchopneumonia. She was treated with ampicillin $2 \mathrm{~g}$ and cloxacillin $2 \mathrm{~g}$ daily, with only partial improvement. During the week after admission signs of heart failure appeared, the heart sounds became softer, and the size of the heart on $x$-ray increased steadily. The electrocardiogram showed low voltage curves with generalized $\mathrm{T}$ wave flattening. A pericardial effusion with tamponade was diagnosed and pericardiocentesis produced $145 \mathrm{ml}$ turbid green pus, with considerable clinical improvement. In view of this, planned open surgical drainage was abandoned. Cultures of the pus were sterile and no organisms were seen on Gram staining. Over the next 5 days twice daily aspiration produced a total of $683 \mathrm{ml}$ pus. After each aspiration I mega unit of penicillin was instilled. Further attempts at aspiration were unsuccessful. She remained febrile, however, and her heart size increased again. Surgical exploration was considered once again but not undertaken since the indication seemed uncertain. Over the next 12 days hepatomegaly, jugular venous engorgement, and pulsus paradoxus reappeared but the heart size remained static. Her chest infection persisted with localized right-sided signs, and the possibility of an inhaled foreign body as the original cause of her illness was entertained. Bronchoscopy was attempted therefore, but cardiac arrest occurred during the procedure and she could not be resuscitated.

At necropsy there was considerable fibrotic thickening of the pericardium producing severe constriction; the

Received 2 August 1972. pericardium was I cm thick; pus was still present in the pericardial sac; the inflammation involved the epicardium; the heart itself was normal.

\section{Case 2}

A 14-month-old girl was admitted to St. Mary's Hospital, Portsmouth, with acute meningitis. The cerebrospinal fluid grew Haemophilus influenzae. She was treated with chloramphenicol I $\mathrm{g}$ and ampicillin $\mathrm{I} \cdot 5 \mathrm{~g}$ daily. The admission chest $x$-ray showed an enormous heart (Fig. I) and the electrocardiogram had low voltage curves with $\mathrm{T}$ wave flattening. There was considerable hepatomegaly and the cardiac impulse was impalpable. Heart sounds were soft but there was no third sound. A pericardial effusion was diagnosed but two attempts at aspiration failed. The signs of meningitis subsided and the cerebrospinal fluid returned to normal but the heart size did not diminish. Nineteen days after admission tamponade became obvious and a further attempt at aspiration produced $250 \mathrm{ml}$ pus. Intrapericardial ampicillin $500 \mathrm{mg}$ were instilled. She was transferred to the Wessex Cardiac and Thoracic Centre. The following day at thoracotomy a further $250 \mathrm{ml}$ pus was removed and a similar quantity of thick inspissated pus was scooped out from behind the heart. The pericardial sac was drained by a wide bore tube with suction. The pus proved to be sterile. After operation ampicillin I $\mathbf{g}$ daily was given and initially she improved, but about one week later oedema, hepatomegaly, jugular venous engorgement, and pulsus paradoxus reappeared. The heart size remained static. Pericardial constriction was diagnosed and operation arranged, but her condition deteriorated so rapidly over the next three days that emergency thoracotomy became essential. At operation (I.K. R.McM) the pericardium was grossly thickened and was constricting the heart. A loculus of thick pus was removed from the posterior part of the pericardial cavity. Extensive pericardial resection was carried out over both ventricles, the phrenic nerve being preserved. The heart failed to expand, however, and it 
FIG. 2 Chest X-ray one year after open pericardial drainage and pericardectomy. 
was observed to be still tightly constricted by a thin layer of epicardium. This was carefully removed from the ventricles between the coronary vessels. Satisfactory expansion of the heart and a rise in blood pressure occurred. She made an uneventful recovery. At follow-up one year later she remains well and the heart appears normal (Fig. 2).

\section{Discussion}

Acute purulent pericarditis is an uncommon disease at any age but particularly so in early childhood (Boyle, Pearce, and Guze, 1961). Gersony and McCracken (1967) were able to find 50 reported cases and added 7 of their own. Keith, Rowe, and Vlad (1967), reviewing the experience at the Hospital for Sick Children, Toronto, discuss 69 patients seen over 40 years. These authors also note that, while the recent incidence of septic pericarditis has declined considerably the mortality has not. Of 56 cases in the 20 years before 1944,50 died $(89 \%)$. Of the 13 cases between 1944 and 1964, 10 died (77\%). Purulent pericarditis is always secondary to infection elsewhere, usually in the lungs. The association with meningitis, as in our second patient, has been documented on several occasions. The commonest causative organism is Staphylococcus pyogenes and Haemophilus influenzae among others may be found.

The management of the acute episode consists of a combination of antibiotic therapy and surgical drainage. Antibiotics alone have not been successful. Gersony and $\mathrm{McC}$ racken found $\mathrm{I} 7$ patients treated in this way, none of whom survived, whereas of 17 other patients treated with antibiotics and drainage only 3 died. Pericardial drainage therefore is essential and even before antibiotics it was frequently successful (Boyle et al., 196I). In our view such drainage should be undertaken by thoracotomy since thick pus frequently loculates or blocks aspiration needles and fine cannulae.
As the acute infection subsided in both our patients, pericardial constriction occurred. Some have doubted the existence of this complication (Gersony and McCracken, 1967) but others (Boyle et al., 196I; Benzing and Kaplan, 1963; Weis and Silber, 196I) recognize, as we do, that constriction may follow pyopericardium within a few weeks and without a pause. It may be easily overlooked in infants and its development can be surprisingly rapid. In our first case this was not appreciated; in the second the speed of deterioration was remarkable. It may be that many deaths in the past have been due to unrecognized acute constriction, and should cardiac failure persist after successful drainage the pericardium should be re-explored.

One point of great interest in our second case was the severity of the fibrotic reaction involving the epicardium. Not until this was extensively removed was constriction relieved.

We are grateful to Dr. R. Brown for kind permission to report Case I, and to Dr. W. B. O'Driscoll for referring the second patient.

\section{References}

Benzing, G., and Kaplan, S. (1963). Purulent pericarditis. American fournal of Diseases of Children, 106, 289.

Boyle, J. D., Pearce, M. L., and Guze, L. B. (I96I). Purulent pericarditis. Review of literature and report of eleven cases. Medicine, 40, II9.

Gersony, W. M., and McCracken, G. H. (1967). Purulent pericarditis in infancy. Pediatrics, 40, 224.

Keith, J. D., Rowe, R. D., and Vlad, P. (1967). Heart Disease in Infancy and Childhood, 2nd ed., p. 978. MacMillan, New York.

Weis, E. I., and Silber, E. N. (196I). Acute constrictive pericarditis. Fournal of Pediatrics, 58, 548.

Requests for reprints to Dr. N. Conway, Southampton Chest Hospital, Oakley Road, Southampton, $\mathrm{SO}_{9} 4 \mathrm{WQ}$. 